\title{
A new equation for the mid-plane potential of power law discs
}

\section{Exact solutions and approximate formulae}

\author{
J.-M. Huré ${ }^{1,2}$, F. Hersant ${ }^{2}$, C. Carreau ${ }^{3}$, and J.-P. Busset ${ }^{1}$ \\ 1 Université de Bordeaux, LAB, 351 cours de la Libération, Talence 33405, France \\ e-mail: [jean-marc.hure; jean-pierre.busset]@obs.u-bordeaux1.fr \\ 2 CNRS/INSU, UMR 5804/LAB, 2 rue de l'Observatoire, BP 89, 33271 Floirac Cedex, France \\ e-mail: franck.hersant@obs.u-bordeaux1.fr \\ 3 La Maurellerie, 37290 Bossay-sur-Claise, France \\ e-mail: cyril.carreau@wanadoo.fr
}

Received 29 February 2008 / Accepted 31 May 2008

\section{ABSTRACT}

\begin{abstract}
Aims. The first-order ordinary differential equation (ODE) that describes the mid-plane gravitational potential in flat finite size discs of surface density $\Sigma(R) \propto R^{s}$ (Huré \& Hersant 2007, A\&A, 467, 907) is solved exactly in terms of infinite series.

Methods. The formal solution of the ODE is derived and then converted into a series representation by expanding the elliptic integral of the first kind over its modulus before analytical integration.

Results. Inside the disc, the gravitational potential consists of three terms: a power law of radius $R$ with index $1+s$, and two infinite series of the variables $R$ and $1 / R$. The convergence of the series can be accelerated, enabling the construction of reliable approximations. At the lowest-order, the potential inside large astrophysical discs $(s \sim-1.5 \pm 1)$ is described by a very simple formula whose accuracy (a few percent typically) is easily increased by considering successive orders through a recurrence. A basic algorithm is given.

Conclusions. Applications concern all theoretical models and numerical simulations where the influence of disc gravity must be checked and/or reliably taken into account.
\end{abstract}

Key words. gravitation - methods: analytical - accretion, accretion disks

\section{Introduction}

Gaseous discs in which the main physical quantities (density, pressure, temperature, thickness, velocity) scale with cylindrical radius as power laws, i.e. "power-law discs", represent an important class of theoretical systems. These are used customary to model accretion in evolved binaries (Shakura \& Sunyaev 1973; Pringle 1981), circumstellar matter (Dubrulle 1992; Edgar 2007), the environment of massive black holes inside active galactic nuclei (Collin-Souffrin \& Dumont 1990; Huré 1998; Semerák 2004) or even the stellar component of some galaxies (Evans 1994; Zhao et al. 1999). In most applications however, power-law discs are truncated either to avoid diverging values at the disc centre (such as density, mass) or in attempting to reproduce the properties of observed discs of finite extension and mass. Although self-similarity is not compatible with the presence of edges, it is generally considered that power laws offer a good description of disc properties in some regions (far from the edges). Note that the presence of sharp edges can be misleading when interpreting observational data (e.g. Hughes et al. 2008). In general, the surface density $\Sigma$ in the outer parts of discs is a decreasing function of the cylindrical radius $R$. Depending on the models, hypotheses, and objects, we have, for instance, $\Sigma \propto R^{-3 / 5}$ in binaries (Shakura \& Sunyaev 1973), $\Sigma \propto R^{ \pm 9 / 20}$ in active galactic nuclei (Collin-Souffrin \& Dumont 1990), $\Sigma \propto R^{-1}$ for a Mestel disc (Mestel 1963), or $\Sigma \propto R^{-3 / 2}$ in circumstellar discs (Piétu et al. 2007). In the context of stationary viscous $\alpha$-discs, a wide range of power-law exponents is allowed since the temperature $T, \Sigma$, and $R$ satisfy the condition (e.g. Pringle 1981):

$\Sigma T R^{3 / 2}=$ const.

while it is $\Sigma \propto R^{-1 / 2}$ in $\beta$-discs (Huré et al. 2001).

The calculus of the gravitation potential of finite-size, powerlaw discs has received little attention yet. Several reasons can be put forward. Solving the Poisson equation or computing the integral of the potential is not a trivial procedure, especially in the presence of edges. It is generally believed that gravity due to low mass discs is unimportant compared with that of a central protostar or black hole, and cannot be probed (see however Baruteau \& Masset 2008). Many studies employ the multi-pole expansion which is known to converge too slowly inside sources to be efficient for the numerical applications (e.g. Clement 1974; Stone \& Norman 1992). Huré \& Hersant (2007) demonstrated that the mid-plane potential of flat power-law discs obeys an inhomogeneous first-order ordinary differential equation (ODE). In this second paper, we discuss the exact solutions of this ODE for the entire physical range (outside and inside the disc) in terms of infinite series. In particular, it is shown that the mid-plane potential is a combination of a power law for the radius $R$ and two series of the variables $R$ and $1 / R$. Since these series converge rapidly inside large discs, it is possible to derive reliable approximations by truncating the series at low orders.

This paper is organised as follows. The ODE for the potential is briefly recalled in Sect. 2 and its formal solution is derived in Sect. 3. In Sect. 4, we express the potential at the two disc 


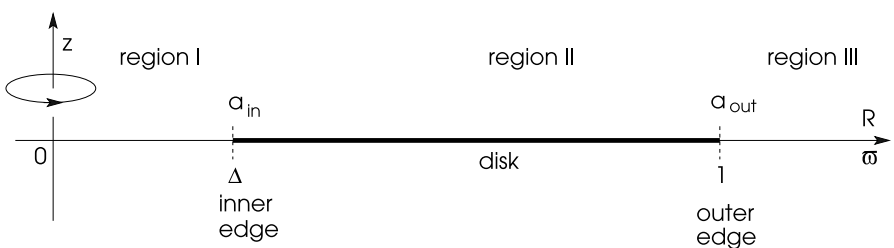

Fig. 1. Configuration for a finite-size flat disc.

edges and consider a few special cases. The inside and outside solutions in the form of series are presented in Sect. 5. In Sect. 6, we analyse the potential in the disc inside in detail, and in particular, the power-law contribution. Since all series involved converge rapidly, we are able to derive reliable approximations for the potential; this is done in Sect. 7. We discuss in Sect. 8 the case of discs with no inner and/or outer edge. The paper ends with a few concluding remarks.

\section{The mid-plane potential in power-law discs from an ordinary differential equation (ODE)}

Following Huré \& Hersant (2007) hereafter Paper I), the midplane potential $\psi$ due to a flat power-law disc satisfies the ODE:

$\frac{\mathrm{d} \psi}{\mathrm{d} \varpi}-(1+s) \frac{\psi}{\varpi}=S(\varpi)$

where $\varpi=R / a_{\text {out }}$ is the cylindrical radius in units of the radius $a_{\text {out }}$ of the disc outer edge, $s$ is the power-law index of the surface density $\Sigma$, namely (generally, $s<0$ in astrophysical discs):

$\Sigma \propto R^{s}$,

and $S(\varpi)$ is the piecewise defined function. Depending on the position in the disc (see Fig. 1), we have:

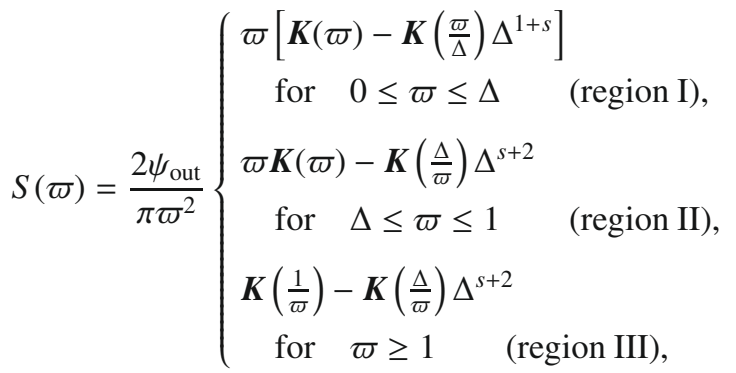

where $\Delta=a_{\text {in }} / a_{\text {out }}<1$ is the axis ratio, $a_{\text {in }}$ is the radius of the inner edge, $\psi_{\text {out }}=2 \pi G \Sigma_{\text {out }} a_{\text {out }}$ is a positive constant, $\Sigma_{\text {out }}$ is the surface density at the disc outer edge, $\boldsymbol{K}$ is the complete elliptic integral of the first kind:

$\boldsymbol{K}(x)=\int_{0}^{\pi / 2} \frac{\mathrm{d} \phi}{\sqrt{1-x^{2} \sin ^{2} \phi}}, \quad 0 \leq x \leq 1$,

and $G$ is the gravitation constant. The above ODE can in principle be solved in the entire radial domain since boundary conditions (both $\psi$ and the associated acceleration $-\mathrm{d}_{\varpi} \psi$ ) are known precisely at $\varpi=0$ and at $\varpi=\infty$ for power law distributions. Although $S$ is singular at the two disc edges (i.e. for $\varpi \in\{\Delta, 1\}$ ), Eq. (1) is far more tractable in computing $\psi$ than the integral form (e.g. Durand 1953):

$\psi(\varpi)=-4 G \Sigma_{\text {out }} a_{\text {out }}^{-s} \int_{a_{\text {in }}}^{a_{\text {out }}} \frac{a^{s+1}}{a+R} K\left(\frac{2 \sqrt{a R}}{a+R}\right) \mathrm{d} a$,

whose integrand is logarithmically singular everywhere inside the disc, or than the Poisson equation, which involves vertical gradients.

\section{Formal solution of the ODE}

A formal solution of Eq. (1) is found by setting (e.g. Rybicki \& Lightman 1979):

$\bar{\psi}(\varpi)=\varpi^{-(1+s)} \psi(\varpi)$,

and

$\bar{S}(\varpi)=\varpi^{-(1+s)} S(\varpi)$.

The exact derivative of $\bar{\psi}$ is:

$\frac{\mathrm{d} \bar{\psi}}{\mathrm{d} \varpi}=\varpi^{-(1+s)}\left[\frac{\mathrm{d} \psi}{\mathrm{d} \varpi}-(1+s) \frac{\psi}{\varpi}\right]$

where we recognise, inside brackets, the function $S$. Therefore, we have:

$\frac{\mathrm{d} \bar{\psi}}{\mathrm{d} \varpi}=\bar{S}(\varpi)$,

whose formal solution for $\bar{\psi}$ is of the form:

$\bar{\psi}(\varpi)=\bar{\psi}\left(\varpi_{0}\right)+\int_{\varpi_{0}}^{\varpi} \bar{S}\left(\varpi^{\prime}\right) \mathrm{d} \varpi^{\prime}$,

Back-substituting $\bar{\psi}$ and $\bar{S}$ from Eqs. (6) and (7), we find the general expression for the mid-plane potential:

$\psi(\varpi)=\varpi^{1+s}\left[\frac{\psi\left(\varpi_{0}\right)}{\varpi_{0}^{1+s}}+\int_{\varpi_{0}}^{\varpi} \frac{S\left(\varpi^{\prime}\right)}{\varpi^{1+s}} \mathrm{~d} \varpi^{\prime}\right]$.

This solution is fully determined in the entire spatial domain or part of it as soon as the potential is known at a given normalised radius $\varpi_{0}$. We observe that, for $s \neq-1, \psi(\varpi)$ is the mixture of a power law of the radius (the first term in the right-handside) with exponent (e.g. Bisnovatyi-Kogan 1975; Evans \& Read 1998):

$p=1+s$,

and a complicated function of the radius $R$ (the definite integral).

In the following, we shall analyse Eq. (11) analytically in terms of infinite series by considering in Eq. (3) the expansion of $\boldsymbol{K}$ over its modulus (e.g. Gradshteyn \& Ryzhik 1965):

$\begin{cases}\boldsymbol{K}(x)=\frac{\pi}{2} \sum_{n=0}^{\infty} \gamma_{n} x^{2 n} & \text { with } 0 \leq x \leq 1, \\ \gamma_{0}=1, & n \geq 1 .\end{cases}$

\section{Potential at the disc edges}

\subsection{Inner edge}

To determine $\psi(\varpi)$ from Eq. (11) for $\varpi \in[0, \infty[$, it is sufficient to calculate the potential at the two disc edges ${ }^{1}$, that is $\psi(\Delta)$ and $\psi(1)$. For this purpose, we use Eq. (5) and define $v=R / a \leq 1$. After some algebra ${ }^{2}$ we find:

$\psi(\Delta)=4 G \Sigma_{\text {out }} \Delta^{1+s} a_{\text {out }} \int_{1}^{\Delta} \frac{\boldsymbol{K}(v)}{v^{2+s}} \mathrm{~d} v$

${ }^{1}$ In contrast with the gravitational acceleration (the gradient of $\psi$ ), the potential is generally finite at the edges.

2 In particular, we use the transformation:

$\boldsymbol{K}\left(\frac{2 \sqrt{x}}{1+x}\right)=(1+x) \boldsymbol{K}(x), \quad 0 \leq x \leq 1$. 


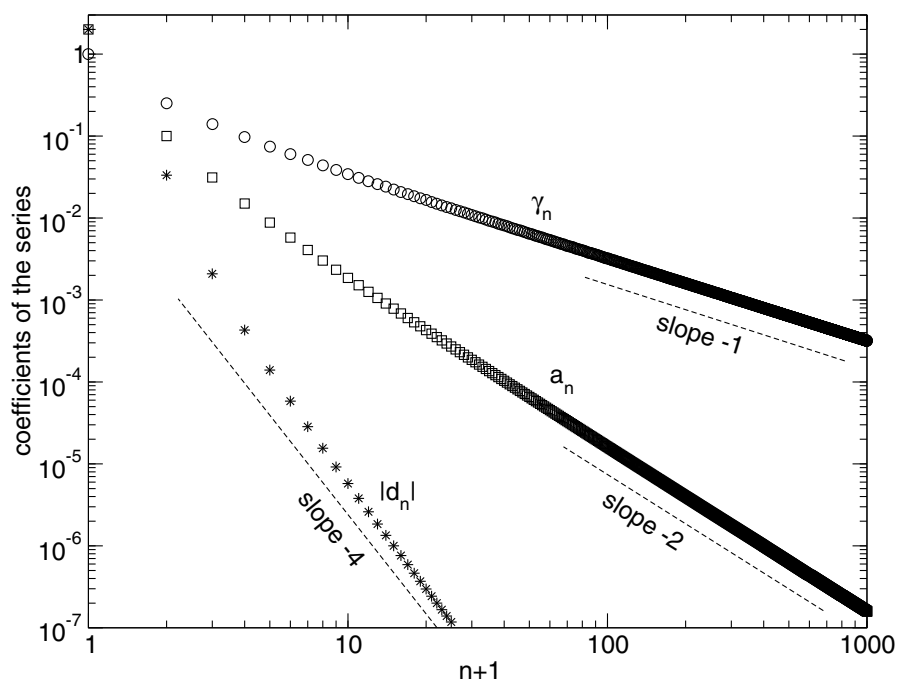

Fig. 2. Coefficient $\gamma_{n}$ versus $n$. Terms $a_{n}$ and $\left|d_{n}\right|$ versus $n$ for $s=-1.5$.

From Eq. (13), the potential at the inner edge is:

$\psi(\Delta)=-\psi_{\text {out }} \Delta^{1+s} \sum_{n=0}^{\infty} I_{n}\left(1-\Delta^{2 n-s-1}\right)$,

where

$I_{n}=\frac{\gamma_{n}}{2 n-s-1}$,

and assuming $\Delta \neq 0$ (see below). As $\gamma_{n} \sim 1 / n$ for large $n$ (see Fig. 2), $\psi(\Delta)$ consists of terms that vary asymptotically like $\sim 1 / n^{2}$. Since $\Delta<1, \psi(\Delta)$ is a converging series.

\subsection{Outer edge}

At the outer edge, the potential is calculated in a similar manner, but using the variable $u=a / R \leq 1$. For $\varpi=1$, Eq. (5) writes ${ }^{1}$ :

$\psi(1)=-4 G \Sigma_{\text {out }} a_{\text {out }} \int_{\Delta}^{1} \boldsymbol{K}(u) u^{1+s} \mathrm{~d} u$.

Replacing $\boldsymbol{K}(u)$ by its series representation yields:

$\psi(1)=-\psi_{\text {out }} \sum_{n=0}^{\infty} J_{n}\left(1-\Delta^{2 n+s+2}\right)$,

where

$J_{n}=\frac{\gamma_{n}}{2 n+s+2}$,

and by assuming $\Delta \neq 0$ (see below). As for $\psi(\Delta)$ and for the same reasons, $\psi(1)$ is also a converging series.

\subsection{Special values of $s$}

If the power law exponent $s$ is such that:

$2 n-s-1=0$

at a certain rank $n \equiv n_{\Delta}$, then $\psi(\Delta)$ must be, in practice, written in a slightly different form. This happens for $s \in \mathcal{E}_{\Delta}$ with:

$\mathcal{E}_{\Delta}=\{-1,+1,+3,+5, \ldots\}$.
Since

$\lim _{q \rightarrow 0} \frac{1-x^{q}}{q}=-\ln x$

for $x>0$ and any $q$, we have:

$\lim _{n \rightarrow n_{\Delta}} I_{n}\left(1-\Delta^{2 n-s-1}\right)=-\gamma_{n_{\Delta}} \ln \Delta$.

Then, if $s \in \mathcal{E}_{\Delta}$, the potential at the disc inner edge is given by:

$\psi(\Delta)=-\psi_{\mathrm{out}} \Delta^{1+s}\left[\sum_{\substack{n=0 \\ n \neq n_{\Delta}}}^{\infty} I_{n}\left(1-\Delta^{2 n-s-1}\right)-\gamma_{n_{\Delta}} \ln \Delta\right]$.

In a similar way, if $s$ is such that:

$2 n+s+2=0$

at a rank $n \equiv n_{1}$, then one term in Eq. (19) must be treated separately. This happens for $s \in \mathcal{E}_{1}$ where:

$\mathcal{E}_{1}=\{-2,-4,-6,-8, \ldots\}$.

From Eq. (23), we have:

$\psi(1)=-\psi_{\text {out }}\left[\sum_{\substack{n=0 \\ n \neq n_{1}}}^{\infty} J_{n}\left(1-\Delta^{2 n+s+2}\right)-\gamma_{n_{1}} \ln \Delta\right]$.

We note that $s$ cannot belong simultaneously to set $\mathcal{E}_{\Delta}$ and to set $\mathcal{E}_{1}$.

\subsection{Cases with $\Delta=0$}

The case $\Delta=0$ occurs i) when the disc has no inner hole (i.e. $a_{\text {in }}=0$ ) but finite size, and/or ii) when the disc has an inner edge but is infinitely extended (i.e. $a_{\text {out }} \rightarrow \infty$ ). In the first case, $\psi(\Delta)$ (denoted $\psi_{\mathrm{c}}$ in Paper I) becomes the potential at the origin of coordinates and has infinite value as soon as $1+s \leq 0$. In the second case, $\psi(1)$ represents the gravitational potential at infinity. It diverges if $s+1 \geq 0$. Figure 3 summarises the ranges of $s$ where the edge surface density, edge potential, and total disc mass are finite. We note that only discs having either i) $a_{\text {in }}=0, a_{\text {out }} \neq \infty$ with $s>0$; or ii) $a_{\text {in }}>0, a_{\text {out }} \rightarrow \infty$ with $s \leq-2$ are physically meaningful since they are characterised by a finite surface density, a finite total mass and a finite potential. Mestel discs do not belong to these categories (e.g. Mestel 1963; Hunter et al. 1984).

\section{Solution of the ODE in the form of series}

We see from Eqs. (3) and (13) that the function $S$ can be easily expressed as a series. We have:

$S(\varpi)=\frac{\psi_{\text {out }}}{\varpi^{2}} \sum_{n=0}^{\infty} \gamma_{n} \times\left\{\begin{array}{lc}\varpi^{2 n+1}\left(1-\Delta^{1+s-2 n}\right) & \text { in region I, } \\ \varpi^{2 n+1}-\varpi^{-2 n} \Delta^{s+2+2 n} & \text { in region II, } \\ \varpi^{-2 n}\left(1-\Delta^{s+2+2 n}\right) & \text { in region III. }\end{array}\right.$

By inserting this general expression into Eq. (11), we find for $s \notin \mathcal{E}_{\Delta} \cup \mathcal{E}_{1}$ and $\Delta \neq 0$ :

$$
\begin{aligned}
& \psi(\varpi)=\frac{\psi\left(\varpi_{0}\right)}{\varpi_{0}^{1+s}} \varpi^{1+s}+\psi_{\text {out }} \sum_{n=0}^{\infty}\left[a_{n}\left(\varpi^{2 n}-\frac{\varpi^{1+s}}{\varpi_{0}^{1+s-2 n}}\right)\right. \\
& \left.+b_{n}\left(\frac{1}{\varpi^{2 n+1}}-\frac{\varpi^{1+s}}{\varpi_{0}^{2 n+2+s}}\right)\right] \text {, }
\end{aligned}
$$




\begin{tabular}{ll|l|l|l} 
i) NO INNER HOLE & -2 & -1 & 0 & exponent $s$ \\
\hline $\begin{array}{l}\text { Inner edge } \\
\text { surface density } \quad \Sigma_{\text {in }}\end{array}$ & & & \\
$\begin{array}{c}\text { Inner edge } \\
\text { potential } \Psi(\Delta)\end{array}$ & & & INFINITE \\
Total disk mass & & & \\
INFINITE & & \\
\end{tabular}

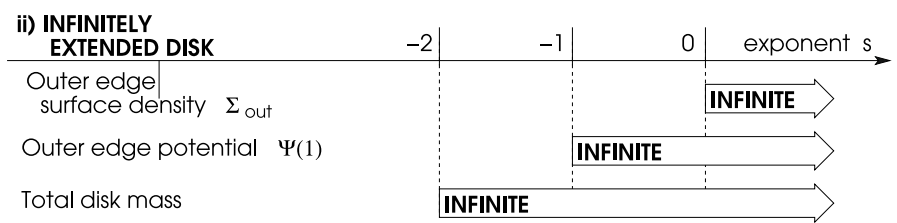

Fig. 3. Ranges of $s$ where the edge surface density, edge potential and total disc mass are infinite.

where the coefficients $a_{n}$ and $b_{n}$ are respectively given by:

$a_{n}=I_{n} \times\left\{\begin{array}{l}1-\Delta^{1+s-2 n} \quad \text { in region I, } \\ 1 \quad \text { in region II, } \\ 0 \text { in region III, }\end{array}\right.$

and

$b_{n}=J_{n} \times\left\{\begin{array}{l}0 \quad \text { in region I, } \\ \Delta^{2 n+s+2} \quad \text { in region II, } \\ \Delta^{2 n+s+2}-1 \quad \text { in region III. }\end{array}\right.$

Since $\psi(\Delta)$ and $\psi(1)$ are available (see Sect. 4), we use $\varpi_{0}=\Delta$ and $\varpi_{0}=1$ to simplify Eq. (30). Using Eqs. (16) and (19), we thus have:

$\frac{\psi(\varpi)}{\psi_{\text {out }}}=A \varpi^{1+s}+\sum_{n=0}^{\infty}\left(a_{n} \varpi^{2 n}+\frac{b_{n}}{\varpi^{2 n+1}}\right)$,

where:

$A=\left\{\begin{array}{l}0, \quad \text { in region } \mathrm{I}, \\ \sum_{n=0}^{\infty} c_{n} \text { in region II, } \\ 0, \quad \text { in region III, }\end{array}\right.$

with:

$c_{n}=-I_{n}-J_{n}$.

We note that $A$ is a function of $s$.

Figure 4 compares the total potential $\psi(\varpi)$ with the power law contribution (i.e. the term $A \varpi^{1+s}$ ) for three typical values of the exponent $s$ in a disc of axis ratio $\Delta=0.1$. We clearly see that, in a finite size disc: i) the gravitational potential is not a powerlaw function of the radius; ii) a power-law contribution is present inside the disc only; and iii) the power law is not the dominant part of the potential. As expected, spatial self-similarity is broken due to edges. We note that, outside the disc (i.e. in regions I and III), the series coincides with the multi-pole expansions.

\section{Potential inside the disc}

The determination of the gravitational potential is usually straightforward outside the distribution where different kinds of expansions are efficient in practice (Kellogg 1929). In contrast, it is problematic inside matter where the classical multi-pole approach fails to converge rapidly (e.g. Clement 1974; Stone \& Norman 1992).

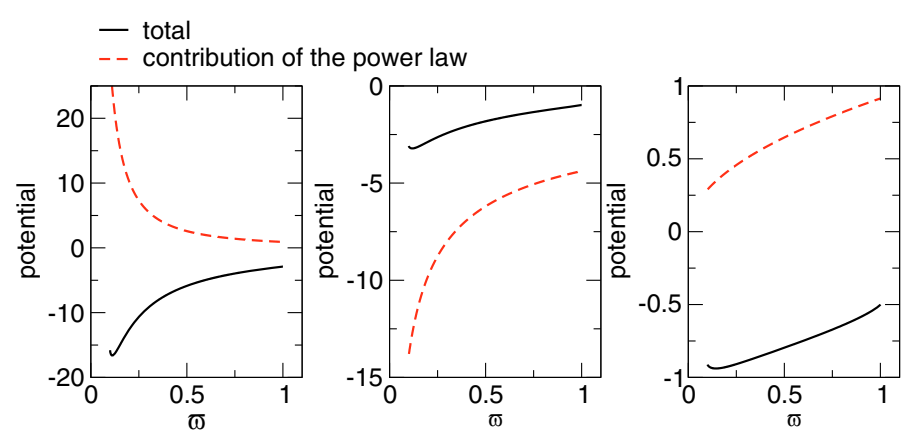

Fig. 4. Potential (plain lines) in a power-law disc with axis ratio $\Delta=0.1$ and $s=\{-2.5,-1.5,-0.5\}$. The contribution of the power law, i.e. the term $A \varpi^{1+s}$ in Eq. (33), is shown in comparison.

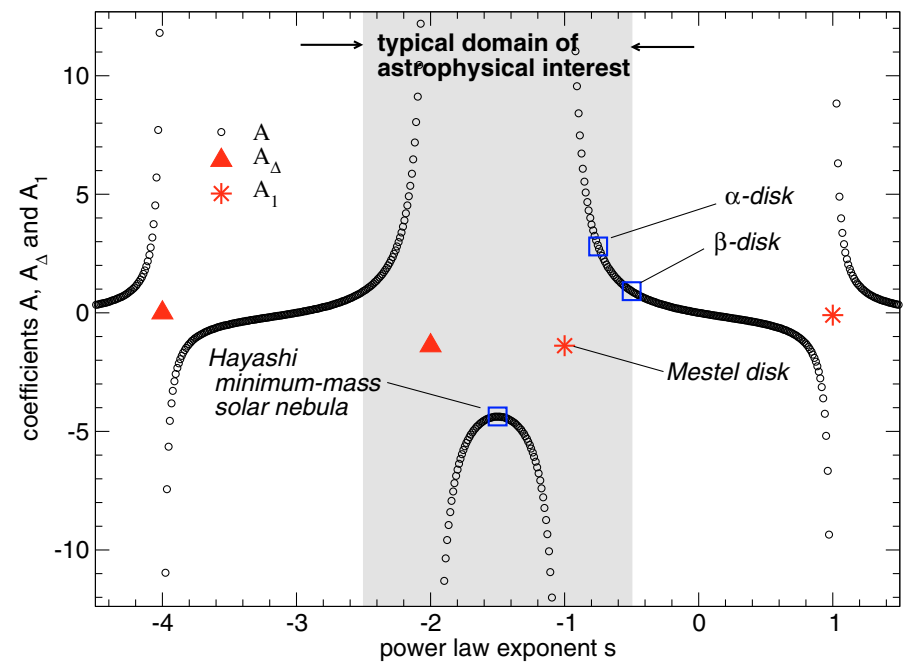

Fig. 5. Coefficients $A$ (open circles), $A_{\Delta}$ (triangles), and $A_{1}$ (stars) versus the power-law exponent $s$ of the surface density. A few remarkable exponents are shown (open squares): the Mestel (infinite) disc with $s=$ -1 (Mestel 1963), the Hayashi model for the solar nebula with $s=-1.5$ (Hayashi 1981), and the $\alpha$ and $\beta$-viscosity discs where $s \approx-0.6$ depending on models (Shakura \& Sunyaev 1973; Collin-Souffrin \& Dumont 1990; Dubrulle 1992; Richard \& Zahn 1999).

\subsection{Converging series}

In region II, we have $b_{n}=J_{n} \Delta^{2 n+s+2}$. If we set:

$X=\frac{a_{\text {in }}}{R} \equiv \frac{\Delta}{\varpi} \leq 1$,

then

$\frac{b_{n}}{\varpi^{2 n+1}}=J_{n} X^{2 n+1} \Delta^{1+s} \leq \Delta^{1+s}$.

Figure 2 shows the term $a_{n}$ versus $n$ for $s=-1.5$ (in this case, $I_{n}=J_{n}$ ). As a consequence, the three series involved in Eq. (33) converge rapidly since i) $I_{n}$ and $J_{n}$ both vary as $1 / n^{2}$ at large $n$;ii) all terms are positive for large $n$; and iii) $\varpi \leq 1$ and $X \leq 1$. This is interesting for the truncation of series and the construction of reliable approximations (see Sect. 7).

\subsection{The coefficient $A$}

The coefficient $A$ is plotted in Fig. 5. It is symmetric with respect to $s=-\frac{3}{2}$ since:

$\frac{c_{n}}{\gamma_{n}}=-\frac{4(4 n+1)}{\left(4 n-2 s^{\prime}+1\right)\left(4 n+2 s^{\prime}+1\right)}$, 
where $s^{\prime}=s+\frac{3}{2}$. For certain integer values of $|s|, A$ is strictly zero. This is in particular the case for $s=-3$ and $s=0$ (see Appendix A). As Eq. (34) shows, $A$ rises as soon as the exponent $s$ is such that either $2 n-s-1$ or $2 n+s+2$ is small (see Sect. 4). Even, if $n=n_{\Delta}$ (or $n=n_{1}$ ), the coefficient $A$ apparently contains a singular term, namely $I_{n_{\Delta}} \varpi^{1+s}$ (resp. $J_{n_{1}} \varpi^{1+s}$ ); however, this singularity exactly cancels with the term $a_{n_{\Delta}} \varpi^{2 n_{\Delta}}$ (resp. $b_{n_{1}} \varpi^{-2 n_{1}-1}$ ). In practice, when $s \in \mathcal{E}_{\Delta}$, Eq. (33) is no longer valid. Instead, we have:

$\frac{\psi(\varpi)}{\psi_{\text {out }}}=\left(A_{\Delta}+\gamma_{n_{\Delta}} \ln \varpi\right) \varpi^{1+s}+\sum_{\substack{n=0 \\ n \neq n_{\Delta}}}^{\infty} a_{n} \varpi^{2 n}+\sum_{n=0}^{\infty} \frac{b_{n}}{\varpi^{2 n+1}}$,

where

$A_{\Delta}=-\sum_{\substack{n=0 \\ n \neq n_{\Delta}}}^{\infty} I_{n}-\sum_{n=0}^{\infty} J_{n}$

Similarly, if $s \in \mathcal{E}_{1}$, the potential writes

$\frac{\psi(\varpi)}{\psi_{\text {out }}}=\left(A_{1}+\gamma_{n_{1}} \ln X\right) \varpi^{1+s}+\sum_{n=0}^{\infty} a_{n} \varpi^{2 n}+\sum_{\substack{n=0 \\ n \neq n_{1}}}^{\infty} \frac{b_{n}}{\varpi^{2 n+1}}$

where

$A_{1}=-\sum_{n=0}^{\infty} I_{n}-\sum_{\substack{n=0 \\ n \neq n_{1}}}^{\infty} J_{n}$

A few values of $A, A_{\Delta}$, and $A_{1}$ are listed in Appendix B. We note that $A_{\Delta}\left(\right.$ or $\left.A_{1}\right)$ differs only from $A$ by the term $I_{n_{\Delta}}\left(\operatorname{resp} . J_{n_{1}}\right)$.

\subsection{Convergence acceleration}

Once $s$ is given, the coefficients $A, A_{\Delta}$, and $A_{1}$ can be easily determined at the required accuracy. It is also possible to improve the convergence rate of the associated series. This accelerates the computation of the coefficients and makes their dependence with the exponent $s$ more explicit. Convergence acceleration is performed by using the properties of the definite integrals of the complete elliptic integrals of the first and second kinds. The demonstration reported in the Appendix $\mathrm{C}$ yields, for $s \notin \mathcal{E}_{\Delta} \cup \mathcal{E}_{1}$ :

$A=\frac{1-6 C}{\pi}-s(s+2) \sum_{n=0}^{\infty} d_{n}-(s+1)(s+3) \sum_{n=0}^{\infty} e_{n}$,

where

$\left\{\begin{array}{l}d_{n}=\frac{I_{n}}{4 n^{2}-1}, \\ e_{n}=\frac{J_{n}}{4 n^{2}-1} .\end{array}\right.$

and $C$ is the Catalan constant (half the area under the function $\boldsymbol{K})$. Numerically, the constant in $A$ is

$\frac{1-6 C}{\pi} \approx-1.4310555380011220$.

Figure 2 shows the coefficient $d_{n}$ for $s=-1.5$ (in this case, $\left.d_{n}=e_{n}\right)$. It follows that, for large $n, d_{n}$ and $e_{n}$ behave like $\sim$ $1 / n^{4}$ asymptotically, and so $A$ approaches its converged value far more rapidly than by means of Eq. (34).

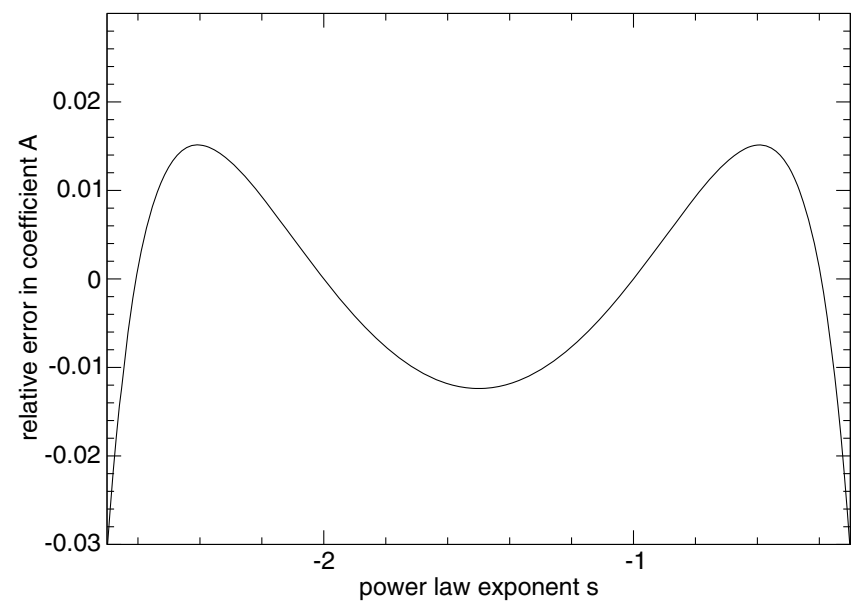

Fig. 6. Relative error in $A$ when computed approximately from Eq. (48).

For $s \in \mathcal{E}_{\Delta}$, we then find:

$$
\begin{aligned}
A_{\Delta}= & \frac{1-6 C}{\pi}+\gamma_{n_{\Delta}} \frac{4 n_{\Delta}}{4 n_{\Delta}^{2}-1}-s(s+2) \sum_{\substack{n=0 \\
n \neq n_{\Delta}}}^{\infty} d_{n} \\
& -(s+1)(s+3) \sum_{n=0}^{\infty} e_{n},
\end{aligned}
$$

instead of Eq. (40), and for $s \in \mathcal{E}_{1}$, this is:

$$
\begin{aligned}
A_{1}= & \frac{1-6 C}{\pi}+\gamma_{n_{1}} \frac{4 n_{1}}{4 n_{1}^{2}-1}-s(s+2) \sum_{n=0}^{\infty} d_{n} \\
& -(s+1)(s+3) \sum_{\substack{n=0 \\
n \neq n_{1}}}^{\infty} e_{n},
\end{aligned}
$$

instead of Eq. (42).

Depending on the exponent $s$ of interest, a good approximation for $A, A_{\Delta}$ or $A_{1}$ can be obtained by considering only the largest terms in the sum, i.e. all terms up to the rank $n \approx \frac{1}{2}(1+s)$ or $n \approx-\frac{1}{2}(s+2)$. For astrophysical discs, $s$ is around -1 meaning that we retain only the first term in Eq. (43). We then find the following approximation:

$A \approx \frac{1-6 C}{\pi}-\frac{s(s+2)}{s+1}+\frac{(s+1)(s+3)}{s+2}$,

whose accuracy is better than $3 \%$ for $-2.7 \lesssim s \lesssim-0.3$ as Fig. 6 shows. For $s \in\{-2,-1\}$, we find from Eqs. (46) and (47):

$A_{\Delta}=A_{1} \approx \frac{1-6 C}{\pi}$

which is in good agreement with the converged value given in Table B.

\section{Approximate formulae inside the disc}

Equations (33), (39) and (41) contain three rapidly converging series that can be truncated to derive reliable approximations for the potential. For $s \approx-1$, only a few terms can be considered (see Sect. 6). Although many truncations are possible, we have noticed that the most accurate approximations of $\psi$ for discs ${ }^{3}$ are obtained provided the coefficient $A$ (or $A_{\Delta}$ or $A_{1}$ depending on $s$ ) takes its converged value. Under these circumstances, the $\mathrm{N}$-order approximation for the potential in region II becomes:

$\frac{\psi_{\mathrm{app} .}^{(N)}(\varpi)}{\psi_{\mathrm{out}}}=A \varpi^{1+s}+\sum_{n=0}^{N}\left(a_{n} \varpi^{2 n}+\frac{b_{n}}{\varpi^{2 n+1}}\right)$,

${ }^{3}$ Here, discs are supposed to be objects of axis ratio $\Delta \lesssim 0.1$. 


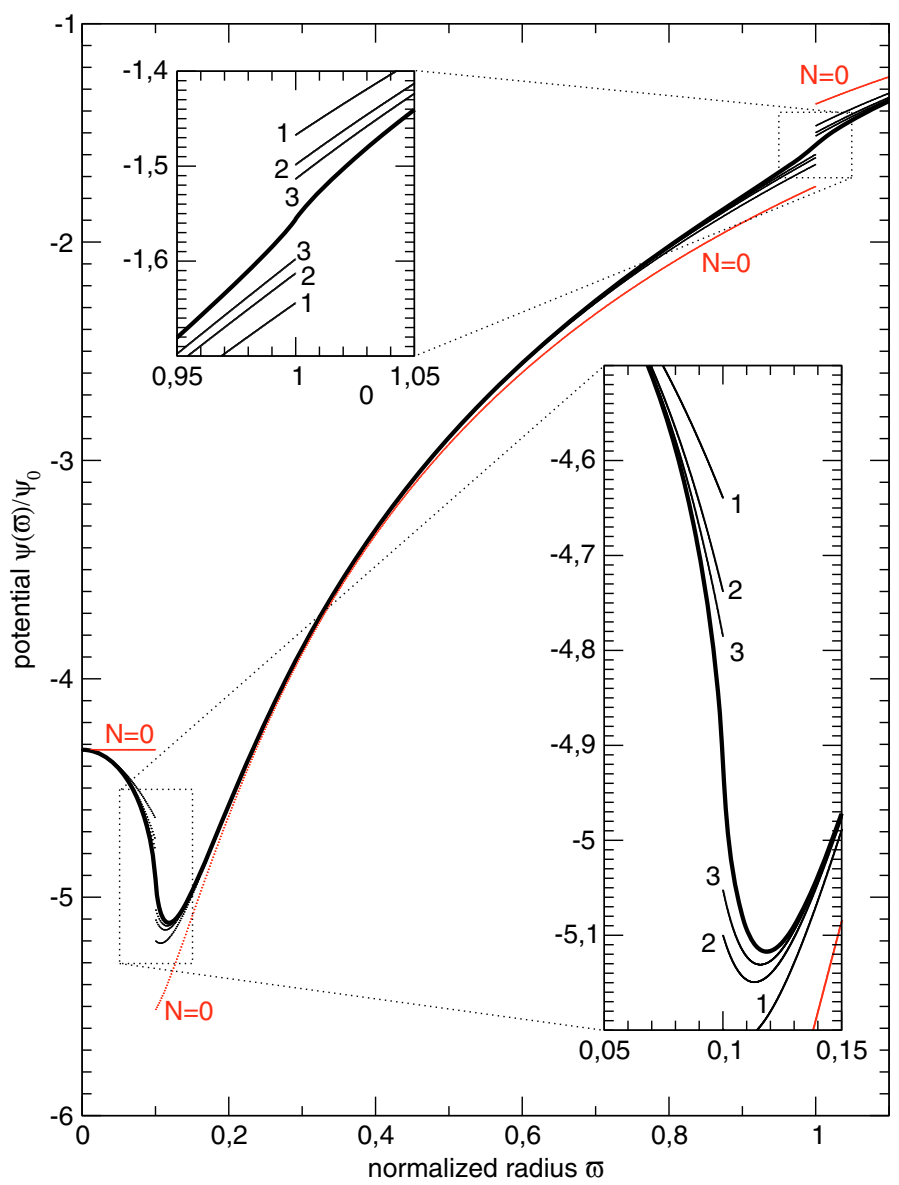

Fig. 7. The exact potential for a power law disc with $s=-1.5$ (thick line) compared to approximate values (thin lines) found from Eq. (50) for $N=0,1,2$ and 3 (i.e. see Eqs. (52), (54), (55), etc.). The largest errors are found around edges.

which is, in its asymptotic limit:

$\lim _{N \rightarrow \infty} \psi_{\text {app. }}^{(N)}(\varpi) \equiv \psi(\varpi)$.

\subsection{Zero-order approximation}

As argued in Sect. 6.3, a reliable formula for the potential in astrophysical discs (for which $s \approx-1.5 \pm 1$ ) is obtained by considering only the terms $a_{0}$ and $b_{0} / \varpi$, in addition to the power law. At the lowest order, we thus have ${ }^{4}$.

$\frac{\psi_{\text {app. }}^{(0)}(\varpi)}{\psi_{\text {out }}}=\left\{\begin{array}{l}\frac{A_{1}+\ln X}{\varpi}+1 \quad \text { if } s=-2, \\ A_{\Delta}+\ln \varpi+X \quad \text { if } s=-1, \\ A \varpi^{1+s}-\frac{1}{s+1}+\frac{\Delta^{1+s} X}{(2+s)} \quad \text { otherwise, }\end{array}\right.$

where, in this case, $A_{1}=A_{\Delta} \approx-1.386$ (see Appendix B). Figure 7 compares this zero-order approximation with the exact potential for typical disc parameters. It follows that the relative deviation $\left|1-\psi_{\text {app. }}^{(0)} / \psi\right|$ does not exceed $10 \%$, the deviation being the largest close to the edges.

It is worth noting that the accuracy remains of the same order if coefficient $A$ is determined by Eq. (48). This is convenient when the explicit dependence of $\psi$ on $s$ is required. Under this

\footnotetext{
4 A careful treatment of the singular cases $s=\{-2,-1\}$ shows that Eq. (52c) yields Eqs. (52a) and (52b).
}

hypothesis, the potential becomes (see note 4):

$$
\begin{aligned}
\psi(R) \approx & -2 \pi G \Sigma_{\text {out }} a_{\text {out }} \times\left\{\frac{1}{1+s}+\left[0.431-\frac{1}{(1+s)(2+s)}\right]\right. \\
& \left.\times\left(\frac{R}{a_{\text {out }}}\right)^{1+s}-\frac{1}{s+2}\left(\frac{a_{\text {in }}}{a_{\text {out }}}\right)^{1+s} \frac{a_{\text {in }}}{R}\right\} .
\end{aligned}
$$

Figure 8 shows the accuracy of this formula in the $(\varpi, s)$-plane. We see that the relative deviation of $\sim 10 \%$ observed previously for $s=-1.5$ holds globally for $s$ roughly in the range ${ }^{5}[-3,0]$. This agrees with the fact that Eq. (48) produces values of $A$ within a few percents for this range of exponents. The deviation can be reduced at the inner and outer edges provided additional terms are included (see below).

\subsection{Higher orders}

If necessary, more accurate expressions are obtained by accounting gradually for following terms (each acting as a smaller and smaller correction). For $N=1$, we have:

$\frac{\psi_{\text {app. }}^{(1)}(\varpi)}{\psi_{\text {out }}}=\frac{\psi_{\text {app. }}^{(0)}(\varpi)}{\psi_{\text {out }}}+\frac{\varpi^{2}}{4(1-s)}+\frac{\Delta^{1+s} X^{3}}{4(4+s)}$,

and for $N=2$, this is:

$\frac{\psi_{\text {app. }}^{(2)}(\varpi)}{\psi_{\text {out }}}=\frac{\psi_{\text {app. }}^{(1)}(\varpi)}{\psi_{\text {out }}}+\frac{9 \varpi^{4}}{64(3-s)}+\frac{9 \Delta^{1+s} X^{5}}{64(6+s)}$,

and so on. We note that Eq. (33) is particularly well suited to numerical computation since $\psi$ can be determined by means of a recurrence procedure. A possible algorithm (not including the treatment of singular cases where $s \in \mathcal{E}_{\Delta} \cup \mathcal{E}_{1}$ ) is proposed in Appendix D.

\section{Discs with no inner/outer edges}

\subsection{Finite disc without inner hole}

If the disc has no inner edge but a finite size (i.e. $a_{\text {in }}=0$ and $a_{\text {out }} \neq \infty$ ), then the ODE is (see Huré \& Hersant 2007):

$S(\varpi)=\frac{2 \psi_{\mathrm{out}}}{\pi \varpi^{2}} \begin{cases}\varpi K(\varpi), & \text { in region II, } \\ \boldsymbol{K}\left(\frac{1}{\varpi}\right), & \text { in region III. }\end{cases}$

It can be verified that the solution is still described by Eqs. (33) and (43), but the coefficients $a_{n}$ and $b_{n}$ are:

$a_{n}= \begin{cases}I_{n}, & \text { in region II } \\ 0, & \text { in region III }\end{cases}$

and

$b_{n}=\left\{\begin{array}{l}0, \quad \text { in region II } \\ -J_{n}, \quad \text { in region III. }\end{array}\right.$

\footnotetext{
5 This range of exponents should be appropriate for most astrophysical applications (see the introduction).
} 

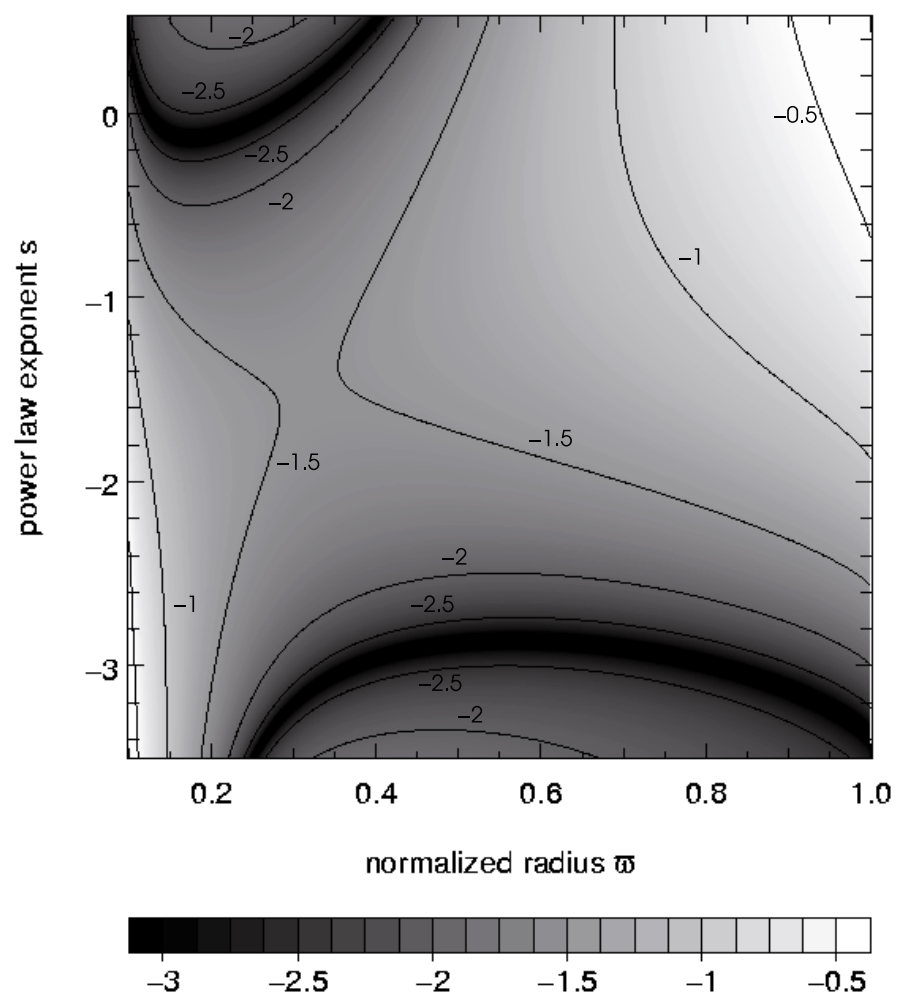

Fig. 8. Contour map showing the decimal logarithm of the relative error in the potential in the $(\varpi, s)$-plane when $\psi$ is approximated by Eq. (53).

\subsection{Infinitely extended disc with inner hole}

If the disc is infinite but has an inner edge (i.e. $a_{\text {in }}>0$ and $a_{\text {out }} \rightarrow \infty$ ), then

$$
\frac{\mathrm{d} \psi}{\mathrm{d} Y}-(1+s) \frac{\psi}{Y}=S(Y)
$$

where

$$
Y=\frac{R}{a_{\text {in }}} \equiv \frac{\varpi}{\Delta}
$$

is the new space variable,

$S(Y)=-\frac{2 \psi_{\text {in }}}{\pi Y^{2}} \begin{cases}Y K(Y), & \text { in region I } \\ K\left(\frac{1}{Y}\right), & \text { in region II, }\end{cases}$

and $\psi_{\text {in }}=2 \pi G \Sigma_{\text {in }} a_{\text {in }}$ is a constant (this is not the potential at the inner edge). The analogue of Eq. (33) is:

$\frac{\psi(Y)}{\psi_{\text {in }}}=A Y^{1+s}+\sum_{n=0}^{\infty}\left(a_{n} Y^{2 n}+\frac{b_{n}}{Y^{2 n+1}}\right)$,

where $A$ is still given by Eq. (34),

$a_{n}= \begin{cases}-I_{n}, & \text { in region } \mathrm{I} \\ 0, & \text { in region II }\end{cases}$

and

$b_{n}= \begin{cases}0, & \text { in region I } \\ J_{n}, & \text { in region II. }\end{cases}$

\subsection{Infinitely extended disc}

If the disc is infinite, the ODE become homogeneous:

$\frac{\mathrm{d} \psi}{\mathrm{d} R}-(1+s) \frac{\psi}{R}=0$,

and the solution is a power law:

$\psi(R)=\psi\left(R_{0}\right)\left(\frac{R}{R_{0}}\right)^{1+s}$,

where $R_{0}$ is some reference radius. In this case only, a selfsimilar surface density can rigorously be associated with a selfsimilar potential. The presence of edges destroys this property. We note that, for $s=-1$ (i.e. Mestel's disc), the derivation of the ODE requires a careful treatment. The integral form, i.e. Eq. (5), gives:

$\psi(R)=-4 G \Sigma_{0} a_{0}\left\{2 C+\frac{\pi}{2}\left[\sum_{n=1}^{\infty} \frac{\gamma_{n}}{2 n}\left(\frac{R}{a}\right)^{2 n}+\ln \frac{a}{R}\right]_{a=a_{\text {out }}}^{a=R}\right\}$.

We then have

$\frac{\mathrm{d} \psi}{\mathrm{d} R}=2 \pi G \Sigma_{0} a_{0} \frac{1}{R}$.

This expression is compatible with Eq. (56a) when $a_{\text {out }} \rightarrow \infty$ (in this case, region III no longer exists and we have $\left.S=\psi_{\text {out }} / \varpi\right)$.

\section{Concluding remarks}

In this paper, we have derived an exact expression for the gravitational potential in the plane of flat power-law discs as a solution of the ODE reported in Paper I. This expression is valid over the entire spatial domain and takes into account finite size effects. Inside the disc (the most difficult case to treat in general), it consists of three terms of comparable magnitude: a power law of the cylindrical radius $R$ with index $1+s$ (where $s$ is the exponent of the surface density) and two series of $R$ and $1 / R$. In terms of convergence, our expression is by far superior to the multi-pole expansion method. Reliable approximations for the potential can be produced by performing fully controlled truncations. We have shown that the potential can be expressed by means of a simple function of $R$ and $s$, which is valid to within a few percents in the range of exponents $-3 \lesssim s \lesssim 0$. This formula should be sufficiently accurate for most astrophysical applications. If necessary, more accurate formulae can be developped by including successive terms. These results should help in investigating various phenomena where disc gravity plays a significant role.

An interesting point concerns the case of discs for which the surface density is not a power-law function. As shown in Paper I, it is easy to reproduce numerically the potential when the profile $\Sigma(R)$ is a mixture of power laws. From an analytical point of view however, the construction of a reliable formula for $\psi$ as compact as the one obtained here is not guaranteed at all. For instance, for an expansion of the form:

$\Sigma(R)=\sum_{s=0}^{M} \alpha_{s} R^{s}$

where $s$ is an integer, each of the $M+1$ series $\left\{a_{n}, b_{n}\right\}$ should be truncated at a rank $N \gtrsim n_{\Delta} \sim(s+1) / 2$ (see Eqs. (33) and (50)), which corresponds to an approximate formula for $\psi$ containing about $2 M(M+1)$ terms. The number of terms to consider can become prohibitively large when several power laws are required.

Acknowledgements. F. Hersant was supported by a CNRS fellowship which is gratefully acknowledged. We thank C. Baruteau. We thank the anonymous referee for valuable comments. 


\section{Appendix A: Vanishing coefficient $\boldsymbol{A}$ for $s \in\{-3,0\}$}

For $s \in\{-3,0\}$, the coefficient is:

$A=-\sum_{0}^{\infty} \gamma_{n}\left(\frac{1}{2 n+2}+\frac{1}{2 n-1}\right)$.

We notice that the first sum is the definite integral of $u K(u)$, which is

$$
\begin{aligned}
\sum_{0}^{\infty} \frac{\gamma_{n}}{2 n+2} & =\sum_{0}^{\infty} \gamma_{n} \int_{0}^{1} u^{2 n+1} \mathrm{~d} u, \\
& =\int_{0}^{1} \mathrm{~d} u \sum_{0}^{\infty} \gamma_{n} u^{2 n+1}, \\
& =\int_{0}^{1} u \mathrm{~d} u \sum_{0}^{\infty} \gamma_{n} u^{2 n} \mathrm{~d} u, \\
& =\frac{2}{\pi} \int_{0}^{1} \boldsymbol{K}(u) u \mathrm{~d} u, \\
& =\frac{2}{\pi}\left[\boldsymbol{E}(u)-\left(1-u^{2}\right) \boldsymbol{K}(u)\right]_{0}^{1}, \\
& =\frac{2}{\pi} .
\end{aligned}
$$

To find the second sum, we compute the definite integral of $K(u) / u^{2}$ in two ways. First, we have by direct integration (e.g. Gradshteyn \& Ryzhik 1965):

$$
\begin{aligned}
\frac{2}{\pi} \int_{0}^{1} \frac{\boldsymbol{K}(u)}{u^{2}} \mathrm{~d} u & =\frac{2}{\pi}\left[-\frac{\boldsymbol{E}(u)}{u}\right]_{0}^{1}, \\
& =-\frac{2}{\pi}\left[\boldsymbol{E}(1)-\lim _{u \rightarrow 0} \frac{\boldsymbol{E}(u)}{u}\right], \\
& =-\frac{2}{\pi}+\frac{2}{\pi} \lim _{u \rightarrow 0} \frac{\boldsymbol{E}(u)}{u}, \\
& =-\frac{2}{\pi}+\lim _{u \rightarrow 0} \frac{1}{u} .
\end{aligned}
$$

Second, by replacing above $\boldsymbol{K}$ by its series representation, we also find:

$$
\begin{aligned}
\frac{2}{\pi} \int_{0}^{1} \frac{\boldsymbol{K}(u)}{u^{2}} \mathrm{~d} u & =\int_{0}^{1} \mathrm{~d} u \sum_{0}^{\infty} \gamma_{n} u^{2 n-2} \\
& =\int_{0}^{1} \mathrm{~d} u\left(\frac{\gamma_{0}}{u^{2}}+\sum_{1}^{\infty} \gamma_{n} u^{2 n-2}\right) \\
& =-\gamma_{0}\left[1-\lim _{u \rightarrow 0} \frac{1}{u}\right]+\sum_{1}^{\infty} \frac{\gamma_{n}}{2 n-1} \\
& =-\gamma_{0}+\gamma_{0} \lim _{u \rightarrow 0} \frac{1}{u}+\left(\frac{\gamma_{0}}{-1}+\sum_{1}^{\infty} \frac{\gamma_{n}}{2 n-1}-\frac{\gamma_{0}}{-1}\right) \\
& =\gamma_{0} \lim _{u \rightarrow 0} \frac{1}{u}+\sum_{0}^{\infty} \frac{\gamma_{n}}{2 n-1} .
\end{aligned}
$$

Since $\gamma_{0}=1$, we have

$$
\sum_{0}^{\infty} \frac{\gamma_{n}}{2 n-1}=-\frac{2}{\pi}
$$

\section{Appendix $B$ : Converged values of coefficient $A, A_{1}$,} and $A_{\Delta}$ for $s \in[-4,1]$

\section{Appendix C: Convergence acceleration for $\boldsymbol{A}$}

The coefficient $A$ is given by a series whose convergence can be accelerated by considering an interesting property of the integral of $\boldsymbol{K}$ (e.g. Gradshteyn \& Ryzhik 1965), namely:

$$
\int_{0}^{1} \boldsymbol{K}(x) \mathrm{d} x=\frac{\pi}{2} \sum_{0}^{\infty} \frac{\gamma_{n}}{2 n+1}=2 C,
$$

where $C$ is Catalan's constant. We can then write

$$
\begin{aligned}
\sum_{n=0}^{\infty} \frac{\gamma_{n}}{1+s-2 n} & =\sum_{n=0}^{\infty} \gamma_{n}\left(\frac{1}{1+s-2 n}+\frac{1}{2 n+1}\right)-\sum_{n=0}^{\infty} \frac{\gamma_{n}}{2 n+1} \\
& =(s+2) \sum_{n=0}^{\infty} \frac{\gamma_{n}}{(2 n+1)(1+s-2 n)}-\frac{4 C}{\pi}, \quad \text { C }
\end{aligned}
$$

where terms vary like $1 / n^{3}$ for large $n$. A second convergence acceleration can be obtained by considering the complete elliptic integral of the second kind:

$\boldsymbol{E}(x)=\int_{0}^{\pi / 2} \mathrm{~d} \phi \sqrt{1-x^{2} \sin ^{2} \phi}, \quad 0 \leq x \leq 1$,

whose definite integral over the modulus $x$ is:

$\int_{0}^{1} \boldsymbol{E}(x) \mathrm{d} x=-\frac{\pi}{2} \sum_{0}^{\infty} \frac{\gamma_{n}}{(2 n+1)(2 n-1)}=\frac{1}{2}+C$.

We then have:

$$
\begin{gathered}
\sum_{n=0}^{\infty} \frac{\gamma_{n}}{(2 n+1)(1+s-2 n)}=\sum_{n=0}^{\infty} \gamma_{n}\left[\frac{1}{(2 n+1)(1+s-2 n)}\right. \\
\left.+\frac{1}{(2 n+1)(2 n-1)}\right]-\sum_{n=0}^{\infty} \frac{\gamma_{n}}{(2 n+1)(2 n-1)} \\
=s \sum_{n=0}^{\infty} \frac{\gamma_{n}}{(2 n+1)(1+s-2 n)(2 n-1)}+\frac{2}{\pi}\left(\frac{1}{2}+C\right) .
\end{gathered}
$$

It follows that:

$$
\begin{aligned}
\sum_{n=0}^{\infty} \frac{\gamma_{n}}{1+s-2 n}= & s(s+2) \sum_{n=0}^{\infty} \frac{\gamma_{n}}{(2 n+1)(1+s-2 n)(2 n-1)} \\
& +\frac{2+s+2 s C}{\pi}
\end{aligned}
$$

The second term in Eq. (34) gives:

$$
\begin{aligned}
\sum_{n=0}^{\infty} \frac{\gamma_{n}}{2 n+s+2}= & \sum_{n=0}^{\infty} \gamma_{n}\left(\frac{1}{2 n+s+2}-\frac{1}{2 n+1}\right) \\
& +\sum_{n=0}^{\infty} \frac{\gamma_{n}}{2 n+1} \\
= & -(s+1) \sum_{n=0}^{\infty} \frac{\gamma_{n}}{(2 n+1)(2 n+s+2)}+\frac{4 C}{\pi} .
\end{aligned}
$$

and so $A=0$ for $s=-3$ and $s=0$. 
Table B.1. Values of $A$ (4th column) computed within the computer precision (double precision; about 16-digit) for different power law exponents $s$ or $s^{\prime}$ (Cols 1 to 3 ) including those relevant for astrophysical applications (see also Fig. 5). Also given are the relative error on the coefficient (5th column) and the number of iterations (6th column) required.

\begin{tabular}{|c|c|c|c|c|c|c|}
\hline$s$ & $-3-s$ & $s^{\prime}$ & Coefficient $A$ & Relative error & Iterations & Comment \\
\hline-4.00 & +1.00 & -2.50 & $-9.65735902799760 \mathrm{E}-02$ & $+3.1 \mathrm{E}-15$ & 6200 & values of $A_{\Delta}$ and $A_{1}$ \\
\hline-3.95 & +0.95 & -2.45 & $-5.08795971306598 \mathrm{E}+00$ & $+1.6 \mathrm{E}-16$ & 4763 & \\
\hline-3.90 & +0.90 & -2.40 & $-2.57897148688964 \mathrm{E}+00$ & $+9.9 \mathrm{E}-17$ & 6289 & \\
\hline-3.85 & +0.85 & -2.35 & $-1.73622548292480 \mathrm{E}+00$ & $+3.9 \mathrm{E}-17$ & 8653 & \\
\hline-3.80 & +0.80 & -2.30 & $-1.30966680644896 \mathrm{E}+00$ & $+2.1 \mathrm{E}-17$ & 10760 & \\
\hline-3.75 & +0.75 & -2.25 & $-1.04923490407066 \mathrm{E}+00$ & $+8.1 \mathrm{E}-17$ & 7977 & \\
\hline-3.70 & +0.70 & -2.20 & $-8.71529232712215 E-01$ & $+1.4 \mathrm{E}-16$ & 7154 & \\
\hline-3.65 & +0.65 & -2.15 & $-7.40760471984439 \mathrm{E}-01$ & $+2.1 \mathrm{E}-16$ & 6696 & \\
\hline-3.60 & +0.60 & -2.10 & $-6.38987239513759 E-01$ & $+2.8 \mathrm{E}-16$ & 6379 & \\
\hline-3.55 & +0.55 & -2.05 & $-5.56193814181879 E-01$ & $+3 \cdot 5 \mathrm{E}-16$ & 6133 & \\
\hline-3.50 & +0.50 & -2.00 & $-4.86319914494768 \mathrm{E}-01$ & $+4.3 \mathrm{E}-16$ & 5926 & \\
\hline-3.45 & +0.45 & -1.95 & $-4.25454571886386 \mathrm{E}-01$ & $+5.2 \mathrm{E}-16$ & 5743 & \\
\hline-3.40 & +0.40 & -1.90 & $-3.70931368062394 \mathrm{E}-01$ & $+6.3 \mathrm{E}-16$ & 5576 & \\
\hline-3.35 & +0.35 & -1.85 & $-3.20839275213352 \mathrm{E}-01$ & $+7.7 \mathrm{E}-16$ & 5420 & \\
\hline-3.30 & +0.30 & -1.80 & $-2.73740796314985 E-01$ & $+9.4 \mathrm{E}-16$ & 5270 & \\
\hline-3.25 & +0.25 & -1.75 & $-2.28500045775376 \mathrm{E}-01$ & $+1.2 \mathrm{E}-15$ & 5124 & \\
\hline-3.20 & $+0.2 \theta$ & -1.70 & $-1.84171894656878 \mathrm{E}-01$ & $+1.5 \mathrm{E}-15$ & 4980 & \\
\hline-3.15 & +0.15 & -1.65 & $-1.39926048201652 \mathrm{E}-01$ & $+2.0 \mathrm{E}-15$ & 4837 & \\
\hline-3.10 & +0.10 & -1.60 & $-9.49911954664950 \mathrm{E}-02$ & $+3.1 \mathrm{E}-15$ & 4694 & \\
\hline-3.05 & +0.05 & -1.55 & $-4.86101640289562 \mathrm{E}-02$ & $+6.3 \mathrm{E}-15$ & 4550 & \\
\hline-3.00 & +0.00 & -1.50 & $-8.70956628336228 \mathrm{E}-15$ & $+3.6 \mathrm{E}-02$ & 4403 & strictly 0 (see Appendix A) \\
\hline-2.95 & -0.05 & -1.45 & $+5.16876888183838 \mathrm{E}-02$ & $+6.4 \mathrm{E}-15$ & 4252 & \\
\hline-2.90 & -0.10 & -1.40 & $+1.07410381098617 \mathrm{E}-01$ & $+3.2 \mathrm{E}-15$ & 4098 & \\
\hline-2.85 & -0.15 & -1.35 & $+1.68286941322651 \mathrm{E}-01$ & $+2.1 \mathrm{E}-15$ & 3937 & \\
\hline-2.80 & $-0.2 \theta$ & -1.30 & $+2.35664501680007 \mathrm{E}-01$ & $+1.6 \mathrm{E}-15$ & 3771 & \\
\hline-2.75 & -0.25 & -1.25 & $+3.11208301380443 E-01$ & $+1.2 \mathrm{E}-15$ & 3596 & \\
\hline-2.70 & -0.30 & -1.20 & $+3.97027142932255 E-01$ & $+1.0 \mathrm{E}-15$ & 3410 & \\
\hline-2.65 & -0.35 & -1.15 & $+4.95854560428368 \mathrm{E}-01$ & $+8.6 \mathrm{E}-16$ & 3212 & \\
\hline-2.60 & -0.40 & -1.10 & $+6.11318936974807 \mathrm{E}-01$ & $+7.4 \mathrm{E}-16$ & 2997 & \\
\hline-2.55 & -0.45 & -1.05 & $+7.48359798181055 \mathrm{E}-01$ & $+6.5 \mathrm{E}-16$ & 2759 & \\
\hline-2.50 & -0.50 & -1.00 & $+9.13893162088934 \mathrm{E}-01$ & $+5.7 \mathrm{E}-16$ & 2486 & \\
\hline-2.45 & -0.55 & -0.95 & $+1.11792019234452 \mathrm{E}+00$ & $+5.1 \mathrm{E}-16$ & 2151 & \\
\hline-2.40 & -0.60 & -0.90 & $+1.37546760819327 \mathrm{E}+00$ & $+4.5 \mathrm{E}-16$ & 1662 & \\
\hline-2.35 & -0.65 & -0.85 & $+1.71019225943660 \mathrm{E}+00$ & $+4.1 \mathrm{E}-16$ & 1335 & \\
\hline-2.30 & -0.70 & -0.80 & $+2.16159222263118 \mathrm{E}+00$ & $+3.7 \mathrm{E}-16$ & 1821 & \\
\hline-2.25 & -0.75 & -0.75 & $+2.80087471242395 \mathrm{E}+00$ & $+3.4 \mathrm{E}-16$ & 1997 & \\
\hline-2.20 & -0.80 & -0.70 & $+3.77063202688011 \mathrm{E}+00$ & $+3.1 \mathrm{E}-16$ & 2058 & \\
\hline-2.15 & -0.85 & -0.65 & $+5.40388067136072 \mathrm{E}+00$ & $+2.8 \mathrm{E}-16$ & 2036 & \\
\hline-2.10 & -0.90 & -0.60 & $+8.70024086898869 \mathrm{E}+00$ & $+2.6 \mathrm{E}-16$ & 1928 & \\
\hline-2.05 & -0.95 & -0.55 & $+1.86592556634413 \mathrm{E}+01$ & $+2.4 \mathrm{E}-16$ & 1681 & \\
\hline-2.00 & -1.00 & -0.50 & $-1.38629436111989 \mathrm{E}+00$ & $+7.2 \mathrm{E}-18$ & 7955 & values of $A_{\Delta}$ and $A_{1}$ \\
\hline-1.95 & -1.05 & -0.45 & $-2.14370823367682 \mathrm{E}+01$ & $+2.1 \mathrm{E}-16$ & 1770 & \\
\hline-1.90 & -1.10 & -0.40 & $-1.14939346514465 \mathrm{E}+01$ & $+1.9 \mathrm{E}-16$ & 2141 & \\
\hline-1.85 & -1.15 & -0.35 & $-8.22454216651923 \mathrm{E}+00$ & $+1.8 \mathrm{E}-16$ & 2399 & \\
\hline-1.80 & -1.20 & -0.30 & $-6.63018820764787 \mathrm{E}+00$ & $+1.7 \mathrm{E}-16$ & 2598 & \\
\hline-1.75 & -1.25 & -0.25 & $-5.71250114438470 \mathrm{E}+00$ & $+1.7 \mathrm{E}-16$ & 2755 & \\
\hline-1.70 & -1.30 & -0.20 & $-5.14024384191494 \mathrm{E}+00$ & $+1.6 \mathrm{E}-16$ & 2878 & \\
\hline-1.65 & -1.35 & -0.15 & $-4.77330268633758 \mathrm{E}+00$ & $+1.6 \mathrm{E}-16$ & 2972 & \\
\hline-1.60 & -1.40 & -0.10 & $-4.54390925876444 \mathrm{E}+00$ & $+1.5 \mathrm{E}-16$ & 3039 & \\
\hline-1.55 & -1.45 & -0.05 & $-4.41737401180816 \mathrm{E}+00$ & $+1.5 \mathrm{E}-16$ & 3078 & \\
\hline-1.50 & -1.50 & +0.00 & $-4.37687923045294 \mathrm{E}+00$ & $+1.5 \mathrm{E}-16$ & 3091 & \\
\hline-1.45 & -1.55 & +0.05 & $-4.41737401180816 \mathrm{E}+00$ & $+1.5 \mathrm{E}-16$ & 3078 & \\
\hline
\end{tabular}


But, from Eq. (C.4), we have:

$$
\begin{gathered}
\sum_{n=0}^{\infty} \frac{\gamma_{n}}{(2 n+1)(2 n+s+2)}=\sum_{n=0}^{\infty} \gamma_{n}\left[\frac{1}{(2 n+1)(2 n+s+2)}\right. \\
\left.-\frac{1}{(2 n+1)(2 n-1)}\right]+\sum_{n=0}^{\infty} \frac{\gamma_{n}}{(2 n+1)(2 n-1)} \\
=-(s+3) \sum_{n=0}^{\infty} \frac{\gamma_{n}}{(2 n+1)(2 n-1)(2 n+s+2)}-\frac{2}{\pi}\left(\frac{1}{2}+C\right),
\end{gathered}
$$

and then:

$$
\begin{aligned}
& \sum_{n=0}^{\infty} \frac{\gamma_{n}}{2 n+s+2}=(s+1)(s+3) \\
& \times \sum_{n=0}^{\infty} \frac{\gamma_{n}}{(2 n+1)(2 n-1)(2 n+s+2)}+\frac{1+s+2(s+3) C}{\pi} .
\end{aligned}
$$

Finally, we have:

$$
\begin{aligned}
A= & \sum_{n=0}^{\infty} c_{n} \\
= & s(s+2) \sum_{n=0}^{\infty} \frac{\gamma_{n}}{(2 n+1)(1+s-2 n)(2 n-1)} \\
& \quad-(s+1)(s+3) \sum_{n=0}^{\infty} \frac{\gamma_{n}}{(2 n+1)(2 n-1)(2 n+s+2)} \\
& +\frac{1-6 C}{\pi} .
\end{aligned}
$$

\section{Appendix D: A basic algorithm}

A possible algorithm is the following (see the text for cases where $\left.s \in \mathcal{E}_{1} \cup \mathcal{E}_{\Delta}\right)$ :

1. disc parameters (see Sect. 2):

- set the inner edge $a_{\text {in }}=? ? ?$

- set the outer edge $a_{\text {out }}=$ ???

- set the outer surface density $\Sigma_{\text {out }}=? ? ?$

- set the power law exponent $s=? ? ?$

$\rightarrow$ compute $\Delta$.

2. power-law coefficient $A$ :

$\rightarrow$ compute $A$ (or $A_{\Delta}$ or $A_{1}$ depending on $s$ ) at the computer accuracy (see Sect. 7); see Eqs. (43), (46) and (47), or use pre-computed converged values (see Appendix B).

3. $\underline{\text { radius }}$

- set the radius $R=? ?$ ? $\rightarrow$ compute dimensionless variables $\varpi$ and $X$ (depending on the region I, II or III).

4. initializations

- set $\gamma_{0}$ to 1 ; see Eqs. (13),

- set $a_{0}$ to $\frac{-1}{1+s}$; see Eq. (31),

- set $b_{0}$ to $\frac{1}{2+s}$; see Eq. (32),

- set $\psi$ to 0 .

5. main loop on $n$

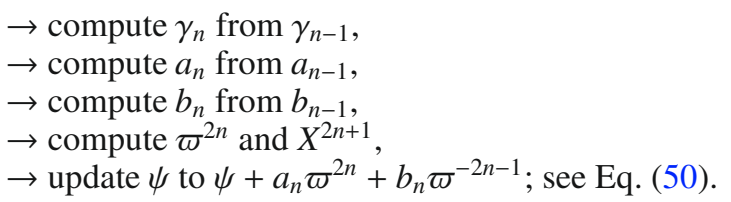

6. power-law contribution

$\rightarrow$ update $\psi$ to $\psi+A \varpi^{1+s}$; see Eq. (50).

7. final potential value

$\rightarrow$ change $\psi$ for $\psi \times 2 \pi G \Sigma_{\text {out }} a_{\text {out }}$.

The loop ends after $N$ steps; the accuracy of the potential value is then given by the next term $(\operatorname{rank} N+1)$.

\section{References}

Baruteau, C, \& Masset, F. 2008, ArXiv e-prints, 801

Bisnovatyi-Kogan, G. S. 1975, SVR Let., 1, 177

Clement, M. J. 1974, ApJ, 194, 709

Collin-Souffrin, S., \& Dumont, A. M. 1990, A\&A, 229, 292

Dubrulle, B. 1992, A\&A, 266, 592

Durand, E. 1953, Electrostatique, Vol. I, Les distributions (Ed. Masson)

Edgar, R. G. 2007, ApJ, 663, 1325

Evans, N. W. 1994, MNRAS, 267, 333

Evans, N. W., \& Read, J. C. A. 1998, MNRAS, 300, 83

Gradshteyn, I. S., \& Ryzhik, I. M. 1965, Table of integrals, series and products (New York: Academic Press), 4th edn., ed. Yu. V Geronimus, \& M. Yu. Tseytlin

Hayashi, C. 1981, Prog. Theor. Phys. Suppl., 70, 35

Hughes, A. M., Wilner, D. J., Qi, C., \& Hogerheijde, M. R. 2008, ArXiv e-prints, 801

Hunter, Jr., J. H., Ball, R., \& Gottesman, S. T. 1984, MNRAS, 208, 1

Huré, J.-M. 1998, A\&A, 337, 625

Huré, J.-M., \& Hersant, F. 2007, A\&A, 467, 907

Huré, J.-M., Richard, D., \& Zahn, J.-P. 2001, A\&A, 367, 1087

Kellogg, O. D. 1929, Foundations of Potential Theory (New-York: Frederick Ungar Publishing Company)

Mestel, L. 1963, MNRAS, 126, 553

Piétu, V., Dutrey, A., \& Guilloteau, S. 2007, A\&A, 467, 163

Pringle, J. E. 1981, ARA\&A, 19, 137

Richard, D., \& Zahn, J.-P. 1999, A\&A, 347, 734

Rybicki, G. B., \& Lightman, A. P. 1979, Radiative processes in astrophysics (New York: Wiley-Interscience), 393

Semerák, O. 2004, Class. Quant. Gravity, 21, 2203

Shakura, N. I., \& Sunyaev, R. A. 1973, A\&A, 24, 337

Stone, J. M., \& Norman, M. L. 1992, ApJS, 80, 753

Zhao, H., Carollo, C. M., \& de Zeeuw, P. T. 1999, MNRAS, 304, 457 